\title{
Research on Framework Model of Applied Professional Courses in Colleges and Universities based on Big Data
}

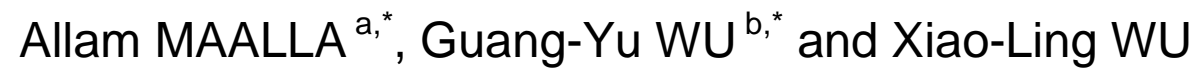 \\ Research Institute of Open Data Commercial Application, School of Information Technology \\ and Engineering, Guangzhou College of Commerce, Guangzhou, China, 511363 \\ aallammaalla@yahoo.com,b975871783@qq.com
}

Keywords: Big Data Technology; Curriculum Framework Model; Corporate Recruitment;

\begin{abstract}
This paper focuses on how to design the professional talent training curriculum framework" more scientific based on big data technology, and leverage big data technology to analyze large-scale online recruitment information and conduct curriculum design and practice. Provide reference ideas for the design and practice of the application-oriented talent training curriculum system for colleges and universities. In the article, the applicationoriented majors, especially the engineering majors, the human understanding and practice of these professional objects that have the characteristics of "periodicity" and "phased". By using the knowledge of web crawler, natural language processing, LDA topic model analysis, knowledge acquisition principle, etc., the data analysis process and implementation method of the talent training curriculum framework oriented by the enterprise recruitment position are proposed.
\end{abstract}

\section{Introduction}

The big data experiment platform with advanced big data integrated experimental system and teaching and training management system provides a virtual simulation environment for practical teaching. Hadoop big data comprehensive experiment system, financial big data industry case lab, cloud training and learning platform, cloud virtual training management platform, cloud application software development and integration platform, etc., can cultivate students' financial business knowledge from different levels and applications. The analysis and design theory and methods of financial information systems have the ability to analyze, design, implement and maintain financial information systems. Cases are available in different scales and difficulty levels, and can be used in all aspects of teaching, including curriculum design, primary school, training, and graduation design. The design of the case corresponds to the curriculum of the university, such as demand analysis, software modeling, object-oriented programming, etc., so the system can be used in conjunction with the course to ensure that students can combine theory with practice, improve learning effects, and provide for different industry applications. Different data analysis and processing modules, through data analysis and processing, companies can clarify the scope of their intelligence and service capabilities, as well as the requirements and solutions implied by the data, and can meet the deep data mining and analysis of different industries. demand. It not only requires talents to have a broader perspective and the ability to solve practical problems, but also has the ability to collect data, analyze data, apply data, and mine data. It is necessary to cultivate financial talents with macroscopic vision and financial market practical problem analysis ability, financial basic theory learning and data application ability parallel, comprehensive and professional, both intellectual and moral. 


\section{Curriculum Structure Analysis Objective}

The relationship between curriculum structures is the basic framework of curriculum system design it can be seen from the design framework of the curriculum system of Figure 1. The application-oriented curriculum framework based on the cultivation of practical ability consists of three main lines, namely, the business process chain, the capability component chain, and the course module chain. Among them, the business process chain can be referenced by experts in the field, and the capacity composition chain and the curriculum module chain content design, involves the exploration and research of the relationship between professional competence and curriculum. Since the form of teaching imparting knowledge is mainly curriculum, the relationship between professional competence and curriculum content is the discussion of the relationship between professional competence and knowledge. According to the hierarchical structure of knowledge, for individuals, knowledge is divided into cognitive and skill layers. The conversion of individual knowledge to cognitive ability is mainly a psychological process, and the skill is often learned by the individual through practice, comprehension and experience in a specific situation. Individuals in this process often need to use external tools to implement them. Correspondingly, in the professional talent training curriculum system, the curriculum can also be divided into cognitive level and skill level. The cognitive level curriculum learning tasks are mainly concepts, principles, and law knowledge. The goal of the skill level course is to master the various skills to solve problems in the knowledge field. In the course framework system, it often shows the use of tools, curriculum design, and graduation design courses.

\section{Job Description" Information}

Job Description information is an important data source for understanding the relationship between "professional competence - curriculum structure "A "post description" field consisting of a longer natural language provides information such as job content and professional skills requirements. This information is a comprehensive reflection of professional knowledge and ability requirements.

For each job category corresponding to the business stage, the "post description" information is often the business capability information related to the position of the category. These job categories, which may be set up to solve a business process phase problem in the field, may also involve work in multiple business process phases in a particular position. Therefore, the relationship between the position and the business stage of the field is a linear correspondence or inclusion relationship shows as follows:

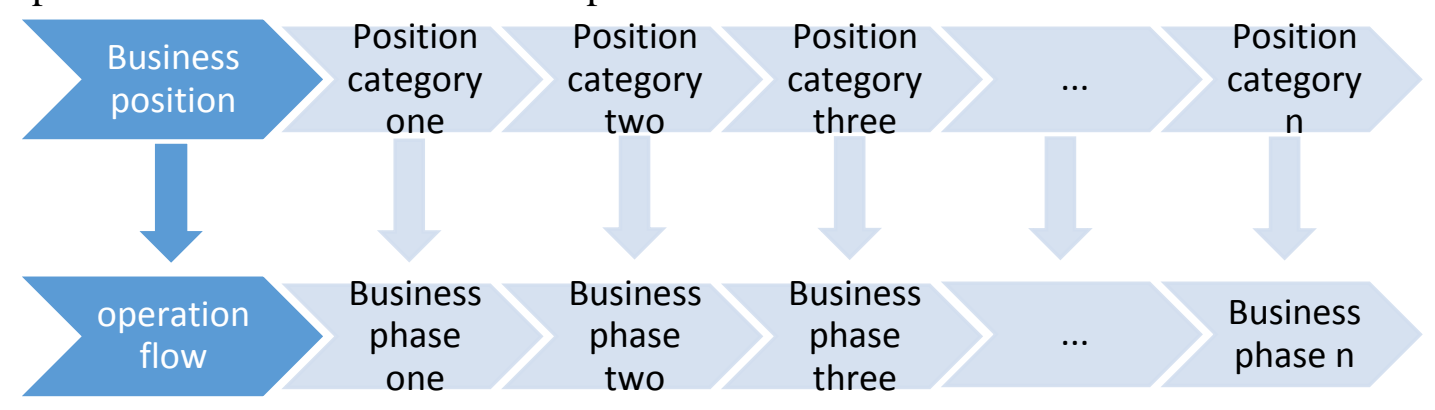

Figure1. Process of the Linear relationship between position and business 


\begin{tabular}{|cl|}
\hline Job category 3 & $\begin{array}{l}\text { : operation flow1 } \\
\text { : operation flow2 } \\
\text { :operation flow3 }\end{array}$ \\
\hline Job category 2 & $\begin{array}{c}\text { : operation flow1 } \\
\text { : peration flow2 }\end{array}$ \\
\hline Job category 1 & -operation flow1 \\
\hline
\end{tabular}

Figure 2. Process of the relationship between position and business

All job description data are analyzed and compared with the job category. The job category analysis can filter out more relevant data sources for the course design and calculate more accurate business phase-capability-course chain relationship data.

\section{Course Structure Data Analysis Process Design}

In summary, the professional competence-course structure derivation process mainly includes two stages: "computation ability theme" and "capability-curriculum structure transformation". The main process of the first phase of the "computational power theme" is as follows:
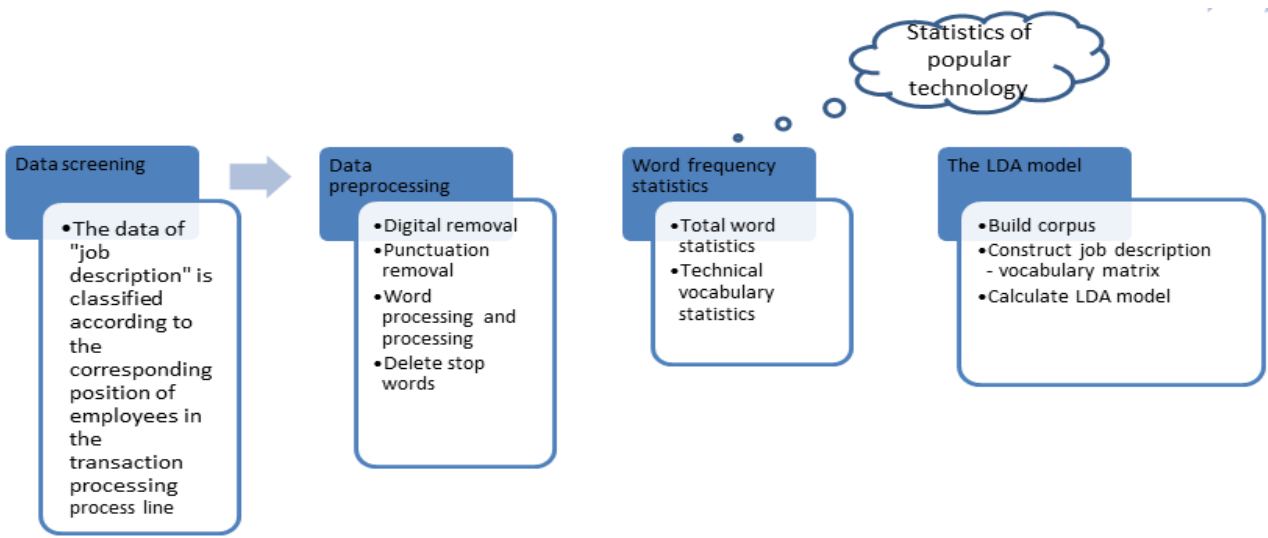

Figure 3. The data analysis process of "ability topic calculation

Firstly, the positions are classified according to the corresponding "business stage" relationship. According to the division result, the "post description" text data of each position category is preprocessed by natural language processing technology. At the same time, the LDA topic model is applied, and after processing, The post description describes the mining of the "capabilities" theme model. In the word frequency statistics, the text related to the "tool" will be analyzed to find out the hot technology, which can provide a reference for the selection of practical teaching tools.

\section{The Specific Implementation of the Curriculum System Framework}

Design and Implementation of Network Recruitment Information Collection Scheme The choice of data processing and analysis methods is determined by factors such as the size, structure, and value density of the data. Data collection mainly involves data sources, data 
acquisition technology, data storage and other work. The data acquisition related scheme is designed as follows:

\section{Data Source Selection}

At present, the three authoritative comprehensive online recruitment websites in China are Carefree, Zhilian Recruitment and ChinaHR.com. Considering the current popularity of each recruitment website, the size of the resume information database, and the distribution of the recruitment industry, Zhilian recruitment is one of the most popular recruitment websites for large and medium-sized recruitment companies. The recruitment information is large, and the recruitment companies cover IT and finance. Services, fast-moving, industrial manufacturing and consulting industries, so this time selected Zhilian recruitment website as the object of collecting recruitment information.

\section{Data Acquisition and Storage Technology}

Data collection uses python3.5 and REDIS in-memory databases to implement distributed crawlers. Multiple computers are used to open multiple crawlers and crawl data. The collected data period is from January 2016 to 2017. It is affected by network instability and website technology adjustment. The accumulated time is 2 months. A total of more than 1.8 million online recruitment information and big data are collected. Job-related recruitment data is about 360,000. When collecting data, first capture the city and job classification, construct a city-job link, grab the job information link of the corresponding page through the city-job link, grab the relevant information of the recruitment information from the recruitment information link, and finally use the MongoDB database technology. Structured storage of fragmented data

\section{Curriculum Structure Analysis}

\section{LDA Model Calculation "Capability" Theme}

Job category division as mentioned earlier, when big data technology is processed in industry domain; the basic process is to determine the basic stages of business objectives, data collection, data extraction transformation and load management, data processing and modeling analysis, data interpretation and application.

According to the professional ability-course structure data analysis process design, the positions need to be classified according to the big data technology application process. It is worth mentioning that the naming of corporate recruitment positions has the problem of nonuniformity. For the same position, there are cases where the company has different naming but the nature of the work is the same. The standardization of these dissident positions is a semi-automated process that requires human involvement.

\section{Curriculum Structure Conversion Implementation}

The LDA theme model calculation results are further processed and refined. The above six topics are: familiar with the network and web page principles; have data capture experience; master Java (data collection language tools); provide job benefits; familiar with data collection protocols; Structure and algorithm. Ability classification "Providing job benefits" is a post salary and welfare issue that can be eliminated. According to the hierarchical structure 
of capabilities, among the remaining five capabilities, cognitive skills are familiar with the principles of web pages, familiarity with data acquisition protocols, and familiarity with data structures and algorithms. The topics that fall into the skill requirements are data acquisition language tools, data capture experience.

\section{Curriculum Design}

Combined with the well-defined cognitive and skill levels, the corresponding teaching content can be further designed to form the knowledge structure framework of the talents in the position. For example, after the data acquisition engineer's ability- Curriculum structure has been converted, the results are as follows:

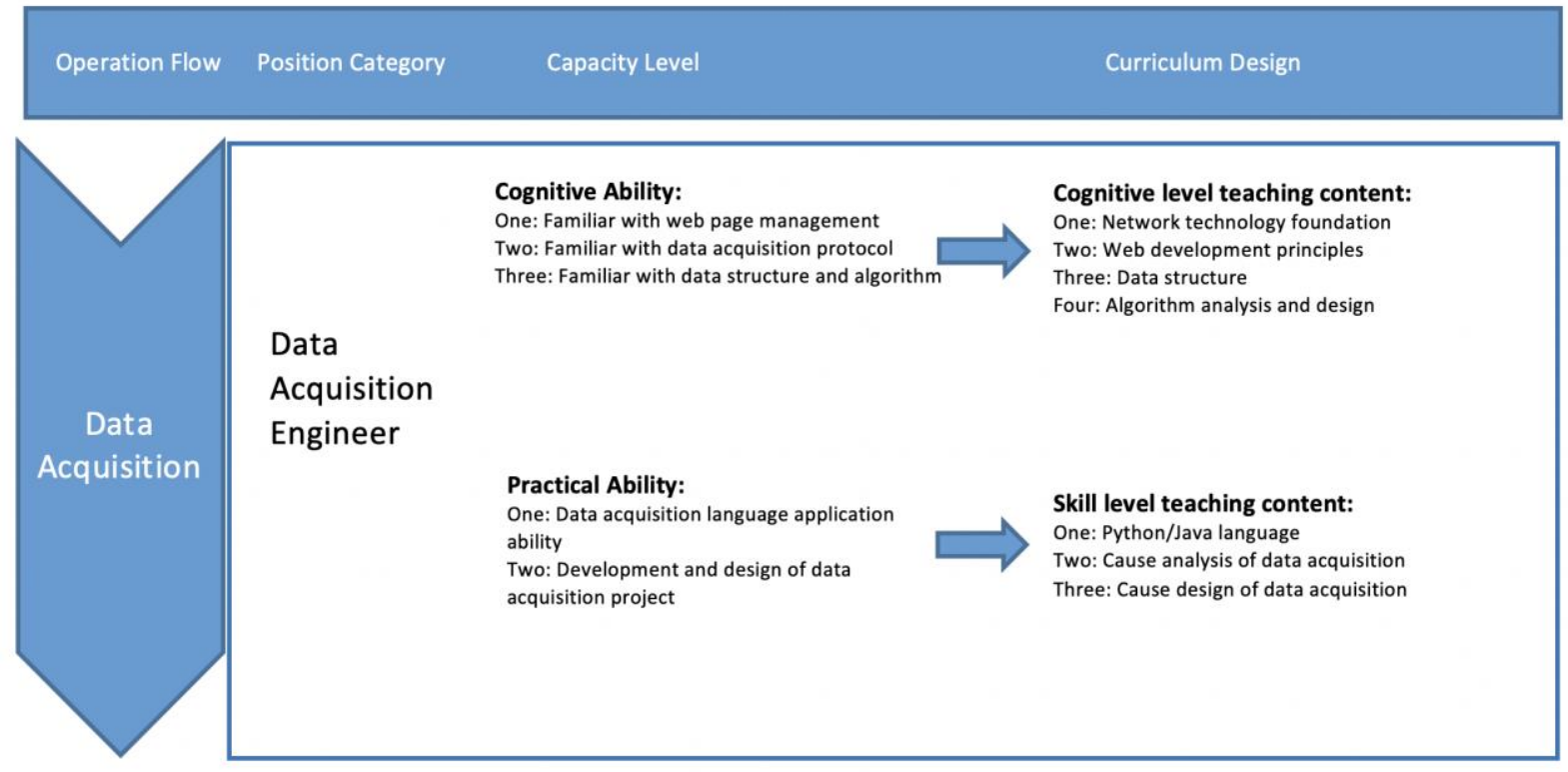

Figure 4. Data Acquisition Engineer "Capability - Curriculum Structure" Relationship Conversion Results

\section{Summary}

This paper applies the big data application technology to the network recruitment data analysis, and puts forward the idea of talent capacity training and course content design according to the job category, which provides a method that can be imitated for the design of college application talent training program. At the same time, it is undeniable that there are professional and applicable differences in the implementation of this method. In this paper, the talent ability-course structure framework design method, compared with history, linguistics and other majors, "engineering" majors such as petroleum engineering, metallurgical engineering, traffic engineering, pharmaceutical engineering, are good reference. The network recruitment information collection task needs to be completed for a period of time. Data processing and analysis is a semi-automated process. This paper focuses on providing practical methods for the design of application-oriented talent training program framework, designing talent corresponding knowledge blocks according to job requirements, focusing on knowledge level training and internal and external knowledge. In summary, the future research direction of this paper can be further improved and explored from the aspects 
of data source collection work operability and how to use big data technology to guide application-oriented personnel training implementation.

\section{Acknowledgement}

This research was financially supported by the 2017 Higher Education Teaching Research and Reform Project of Guangdong Province, "The Big Data Talent Cultivation Practice Teaching System of Business Schools" (Project No. 2017SJJXGG01), and Characteristic Key Subject of E-commerce Construction Project of Guangzhou College of Commerce Foundation, (Project No.TSZDXK201601).

\section{References}

[1] Maalla A, Zhou C, Guang-Yu W U. The Construction of Heterogeneous Platform of Unified Service System Based on Cloud Computing [J]. 2017(cmee). DOI: $10.12783 / \mathrm{dtcse} / \mathrm{cmee} 2017 / 20056$.

[2] DeNisi, A.S. and R.W. Griffin, "Human Resource Management"[M]. 2015, New York: Houghton Mifflin Company.

[3] Jiang Z.li. Improving the Teaching and Learning Level of Computing Subjects Based on Ability Cultivation [J]. University Teaching in China, 2014, (8): 35-37.

[4] Computer Science and Technology Teaching Steering Committee of the Ministry of Education. Public core knowledge system and curriculum of computer science and technology in colleges and universities [M]. Beijing: Tsinghua University Press, 2012.

[5] Chen Tao, Wang Pingshui. Research on the Ways of Cultivating Innovative Talents of Computer Majors in Internet + Financial Colleges [J].Fujian Computer .2015, (11):44-46.

[6] Ranjan, J., "Data Mining Techniques for better decisions in Human Resource Management Systems" [J]. International Journal of Business Information Systems, 2015, 3(5): 464-481.

[7] Zhang Zhigang, Zhao Liqiang. Gao Tianying. Research on training program of computer science professionals for big data applications [J], Tianjin Education. 2017, (1): 32-33.

[8] Zhang Fan. Thinking on the cultivation of computer professional application talents in the direction of mobile internet [J]. Science and Technology Information. 2015, (24): 129-130. 